\title{
Does diabetic early kidney damage alter renal elasticity?: An ultrasound based 2D Shear wave elastography study
}

\author{
Nesrin Gündüz ${ }^{1}$, Aysenur Buz ${ }^{1}$, and Adnan Kabaalioglu² \\ ${ }^{1}$ Istanbul Medeniyet University Faculty of Medicine \\ ${ }^{2}$ Koc University
}

June 3, 2021

\begin{abstract}
Objectives: Kidney damage caused by type-II diabetes mellitus (DM-II) can disturb renal elasticity. There is a paucity of data whether early kidney damage causes kidney stiffening. This comparative study aimed to assess kidney elasticity in patients with DM-II with or without moderate albuminuria using ultrasound based 2D shear wave velocity (2D-SWV). Methods: For this prospective single center study, a sample size of at least 32 was computed. Overall 57 cases (40 DM-II subjects with no chronic kidney disease and 17 age and sex matched healthy controls) were included. The DM-II subjects comprised moderate albuminuria $(\mathrm{n}=22)$ and no-albuminuria $(\mathrm{n}=18)$ subgroups. Bilateral renal parenchymal 2D-SWV values were separately measured from upper, mid and lower portions. Groups were compared with T test or Mann-Whitney-U test as appropriate. Inter-reader agreement was assessed by intra-class correlation coefficient (ICC). Results: The median age [55.5 (50-62) vs 55 (48.5-59.5) years, respectively, $\mathrm{p}=0.48]$ and gender rates $[18(45 \%)$ vs $10(58.8 \%)$ females, respectively, $\mathrm{p}=0.34]$ did not differ between DM-II and control groups. The average and individual portion 2D-SWV values were also similar ( $\mathrm{p}>0.05$ for all). The average 2D-SWV values were also similar between the albuminuria vs no-albuminuria subgroups. The interobserver agreement was good (ICC: $0.66,95 \%$ CI: 0.19-0.88, $\mathrm{p}=0.006$ ). Conclusion: The kidney elasticity does not seem to be disturbed in patients with diabetes and a preserved eGFR with or without moderate albuminuria.
\end{abstract}

\section{INTRODUCTION}

Chronic kidney disease (CKD) is defined as sustained kidney damage or decrease of estimated glomerular filtration rate (eGFR) to less than $60 \mathrm{ml} / \mathrm{min}$ per $1.73 \mathrm{~m}^{2}$ body surface area lasting for [?]3 months (1). Diabetes mellitus (DM) is one of the most common causes of kidney damage and dysfunction (2). DM-II causes endothelial dysfunction and increased permeability to proteins (3). Moderate albuminuria, defined as urinary albumin to creatinine ratio (uACR) between $30-300 \mathrm{mg} / \mathrm{g}$, is one of the most commonly used biomarker of early renal injury.

The most common noninvasive imaging tool for the assessment of renal diseases is ultrasonography (US) with renal size, cortical echogenicity and cortical thickness being used as markers of CKD $(4,5)$. Although these conventional US markers are well correlated with eGFR and severity of albuminuria, they can be recognized relatively late in the disease course where advanced damage to kidneys has already occurred (6). It is well recognized that histological changes in kidneys start before a measurable decline in eGFR and conventional US findings have occurred (3). Typical histological changes in type-II DM (DM-II) include glomerular changes which are related to proteinuria (7). Interstitial fibrosis occur in the later stages, and are associated with a decline in GFR and progressive deterioration of tissue elasticity and increased renal stiffness $(3,6)$. Hence combination of moderately increased uACR with preserved eGFR can be considered as a surrogate marker of early kidney damage in patients with DM-II.

The so called gold standard method to detect early glomerular changes is biopsy (8) which is invasive and 
inappropriate for prospective serial follow-up (9). Moreover, biopsy sensitivity remains problematic due to potential sampling errors $(8,10)$. Hence, elastographic methods have been increasingly investigated to assess kidney stiffness including magnetic resonance imaging (11) and US (6, 9, 12, 13). Among US shear-wave elastography methods, 2D shear-wave velocity (2D-SWV) has emerged as a relatively novel method to assess tissue stiffness and has been reported to provide comparable results as compared to transient elastography point shear-wave elastography $(14,15)$. There is lack of evidence showing disturbances in renal elasticity caused by early diabetic renal damage. This study aims to compare the renal elasticity DM-II patients with preserved eGFR and healthy controls using US 2D-SWV.

\section{METHODS}

This prospective-observational single center study was conducted in accordance with the Declaration of Helsinki and Institutional Research Ethics Committee approved our study design. Written informed consent was obtained from all participants.

\section{Study design and participants}

Sample size calculation for mean SWV's based on a previous publication revealed a total of 30 patients to be enrolled (at least 15 healthy and 15 healthy controls). However, since we pre-planned to stratify DM-II patients in two groups, doubling of sample size of DM-II subjects was considered appropriate. The study was performed in our tertiary institution on a three consecutive months period. Overall 57 subjects including 40 outpatients with DM-II and 17 age and sex matched healthy controls were enrolled. DM-II patients were recruited from internal medicine or nephrology outpatient clinics and control subjects were asked to participate in the study by in-hospital advertisements.

Patients with DM-II were divided into two groups according to the presence of moderately elevated uACR [Groups with (Alb+) and without (Alb-) albuminuria]. During initial scanning of subjects with DM-II, those with more than mild reduction in eGFR (i.e. less than $<60 \mathrm{ml} / \mathrm{min} / 1.73 \mathrm{~cm} 2$ ), more than moderate proteinuria (urine ACR $>300 \mathrm{mg} / \mathrm{g}$ ) or already being treated for proteinuria, other confirmed diabetic organ complications, any systemic disease with a potential of renal involvement, history or symptoms of cardiovascular diseases, or common risk factors for vascular diseases (i.e. hypertension, smoking, dyslipidemia) were disqualified from the study. The uACR was measured from early morning urine samples, in at least 2 occasions of at least 3 months apart. The eGFR was calculated with 2009 CKD-EPI formula using serum creatinine value measured in at least 2 occasions of at least 3 months apart (16).

None of patients were not scheduled for elastography assessment by the clinician but were imaged for research purposes. Moreover, a scanning conventional kidney US was performed after initial recruitment and those with abnormal findings (renal tumor, hydronephrosis, congenital anomaly, signs of acquired renal disease) were excluded.

\section{US shear-wave elastography technique}

2D-SWV based US elastography was performed using Toshiba Aplio i800 (Canon Medical systems, Japan) ultrasound system with an ultra-wideband convex transducer (i8CX1). The images were obtained while the patients lying in the lateral decubitus position with breath-hold during data acquisition. The sample box was placed in the renal cortex of the upper, middle and lower portions of each kidney, respectively (Fig. 1). A fixed region-of-interest (ROI) size was chosen for each measurement. The 2D-SWV was measured 4-6 times for each renal portions and the mean of the measurements were recorded accordingly. The mean 2D-SWV values of the whole right and left kidneys were also calculated by averaging the values of three renal portions. A second radiologist repeated the right kidney average 2D-SWV measurements in randomly selected 10 healthy subjects on the same day.

\section{Statistical Analysis}

Statistical analysis was performed with SPSS 19.0 (IBM SPSS Inc. Chicago, IL, USA). The normality of continuous variables were analysed by Shapiro-Wilk test. Descriptive statistics were reported as mean 
with standard deviation for continuous variables with normal distribution and as median with 25th-75th percentiles for those without. Categorical variables were reported as frequencies with percentages. Independent two-group comparisons for continuous variables without normal distribution, were tested using Mann-Whitney U test. For continuous variables with a normal data distribution, unpaired T-test was used. The proportions were compared between the groups using Pearson's Chi-square test in case the assumptions were met. Otherwise, Fisher's exact test was used. Due to biological relationship between aging and loss of renal function, a logistic regression analysis model including age and 2D-SWV of relevant portions (those with intergroup $\mathrm{p}<0.05)$ was constructed in order to predict the presence of Alb+ status. Significance level was accepted at $\mathrm{p}<0.05$ for all statistical analyses.

A subgroup of a previous study included elasticity assessment of patients resembling our study population (17). Based on the mean SWV's taken from that study, we performed an a priori sample size calculation using the prespecified alpha level of 0.05 and a beta level of 0.20 and an expected effect size of 0.20 (a relative $\% 20$ less SWV in healthy subjects as compared to diabetics). A total sample size of 28 was computed, we aimed to exceed 14 patients to be enrolled in each group. Moreover since it was planned to stratify the DM-II patients into two groups, the sample size of DM-II group was doubled.

\section{RESULTS}

Overall 57 patients including 40 with DM-II and 17 age and sex matched healthy controls were included in the study. The median age [55.5 (50-62) vs 55 (48.5-59.5) years, respectively, $\mathrm{p}=0.48]$ and gender rates $[18$ $(45 \%)$ vs $10(58.8 \%)$ females, respectively, $\mathrm{p}=0.34]$ did not differ between the groups. Inter-rater reliability analysis revealed good agreement for 2D-SWV (ICC 0.66, 95\% CI: 0.19-0.88, p=0.006).

Patients with DM-II were further divided into two subgroups according to uACR. Twenty two (55\%) of DM-II cases had moderately increased uACR and 18 (45\%) had no albuminuria. The age [55.5 (49.5-63) vs 56 (51-60.25) years, respectively, $\mathrm{p}=0.71]$ and gender rates [11 (50\%) vs 7 (38.9\%), females, respectively, $\mathrm{p}=0.48]$ were similar between Alb + and Alb- subgroups.

\section{Comparison of 2D-SWV between DM-II vs Healthy subjects}

The average 2D-SWV of right [1.89 (1.73-2.18) vs $1.99(1.83-2.38) \mathrm{m} / \mathrm{s}$, respectively, $\mathrm{p}=0.18]$ or left [1.91 $(1.66-2.13)$ vs $1.95(1.58-2.1) \mathrm{m} / \mathrm{s}$, respectively, $\mathrm{p}=0.92]$ kidneys did not differ between the DM-II group and healthy controls. Moreover, the 2D-SWV values of the individual (upper, mid, and lower) portions were also similar between both groups ( $\mathrm{p}>0.05$ for all) for both kidneys (Table 1).

\section{Comparison of 2D-SWV between Alb+ vs Alb- subgroups}

The average 2D-SWV of right [2 (1.75-2.28) vs $1.83(1.71-2.06) \mathrm{m} / \mathrm{s}$, respectively, $\mathrm{p}=0.32$ ] or left [1.99 (1.69$2.24)$ vs 1.76 (1.55-2.1), respectively, $\mathrm{p}=0.13$ ] kidneys did not differ between the Alb+ and Alb- subgroups. Only the 2D-SWV values of the upper pole of right kidney [2.02 (1.46-2.43) vs $1.83(1.63-2.11) \mathrm{m} / \mathrm{s}$, respectively, $\mathrm{p}=0.04]$ and mid portion of left kidney [1.96 (1.64-2.23) vs $1.54(1.35-2) \mathrm{m} / \mathrm{s}$, respectively, $\mathrm{p}=0.03]$ were significantly higher in Alb+ subgroup. However, the 2D-SWV of the remaining portions of both kidneys did differ between both subgroups (Table 2).

\section{Effect of gender on 2D-SWV}

All the 2D-SWV's of the individual portions and the average 2D-SWV's of both kidneys were similar between males and females ( $\mathrm{p}>0.05$ for all).

\section{Effect of age on 2D-SWV}

A weak positive correlation between age and average 2D-SWV's of left kidneys was found (correlation coefficient: $0.29, \mathrm{p}=0.03)$. However no correlation was present between age and average 2D-SWV's of right kidneys (correlation coefficient: 0.054, $\mathrm{p}=0.69$ ).

\section{Prediction of albuminuria}


Since 2D SWV's of right kidney upper pole and left kidney mid portion appeared to significantly differ between mAlb+ and mAlb- subgroups and age showed some correlation with SWV, logistic regression analysis was performed. The first model included age and left mid portion SWV and did not reveal a significant predictor of albuminuria status. The second model included age and the right upper pole SWV, again with negative results (Table 3).

\section{DISCUSSION}

The main finding of current study is that, a significant change in renal cortical elasticity was not found between patients with DM-II versus healthy subjects or in albuminuria versus no-albuminuria subgroups.

DM-II causes microstructural changes in renal parenchyma before a significant drop in eGFR occurs (3). A moderately increased uACR indicates presence of kidney damage and is associated with future risk of progression to kidney dysfunction and cardiovascular complications independent of eGFR (16). We recognize that histological changes can be present in a number of subjects with DM-II before pathologic amount of urinary albumin can be detected $(3,7)$. Hence, one may argue that biopsy is required for definitive confirmation of typical diabetic changes in glomeruli (8). However, biopsy is not performed routinely in all cases with DM-II and mostly reserved for certain indications (9). In the landmark study by Fioretto P et al, microalbuminuric DM-II patients with normal eGFR underwent kidney biopsy for research purposes rather than for a clinical indication (18). They found histopathological changes (either typical glomerular or atypical tubulointerstitial and vascular changes), in $70 \%$ of DM-II subjects. That was the basis that a moderately elevated uACR in the setting of preserved eGFR was used as representative of early diabetic renal injury in the current study where biopsy was not available. We have found that, the early diabetic histologic changes in kidneys do not necessarily translate into disturbed elasticity, as we have not observed a difference in SWV values between albuminuria and no-albuminurai subjects.

Conventional kidney US findings alone are inadequate for early recognition of kidney damage in most cases. Indeed the typical US findings of CKD represent late changes (4-6), which are already irreversible. Moreover the conventional US findings of CKD are not specific for diabetic nephropathy and diverse etiologies share the common imaging changes. Elastography, on the other hand, has emerged as a tool for assessment of increased tissue stiffness caused by damages to organs including liver, breast, thyroid, prostate and kidney. The relatively novel US shear-wave elastography method was used to assess kidney stiffness in our study. 2D-SWV measurement by this technique has provided comparable results to previously described methods including transient elastography and acoustic radiation force imaging in depicting liver fibrosis (15). Although SWV values are positively correlated with amount of fibrosis in liver diseases (19), it is negatively correlated with CKD stages and eGFR $(6,12)$. The underlying mechanism of the inverse relationship between stiffness and eGFR in patients with CKD remains unclear. Altered renal perfusion has been proposed to affect stiffness measurements and explain this paradox (20). The ARFI derived SWV has been found to be higher in patients with DM-II versus healthy controls in one study (21). Moreover others revealed increased SWV in DM-II induced early kidney damage but no CKD (i.e. eGFR still more than $60 \mathrm{ml} / \mathrm{min} / 1.73 \mathrm{~m} 2$ ) versus diabetic CKD (i.e. eGFR already less than $60 \mathrm{ml} / \mathrm{min} / 1.73 \mathrm{~m} 2$ ) (17). Interestingly our results does not support these findings. The whole kidney average SWV's were not found to be increased neither in DMII vs healthy controls nor in mAlb+ vs mAlb- subjects in current study. The contradiction between both studies can be explained by the differences in study populations. Goya et al (17) did not report any exclusion criteria in terms of comorbidities that may interfere with elasticity results (hypertension, systemic diseases, other diabetic organ complications, risk factors for vascular diseases). On the other hand our population comprised a very selective group that presence of a confounder of elasticity would be very unlikely. Another factor that may influence the results is the sonographic technique used for elasticity assessment. We used 2D-SWV measurement, whereas others used the ARFI method.

The left middle and right upper portions' elasticity seemed to be selectively disturbed in mAlb+ cases at univariate comparison. One may argue that this may be attributable to the potentially asynchronous severity of changes in different portions of kidneys $(8,10)$. Indeed, heterogeneous renal histopathologic changes may occur in albuminuric patients with DM-II $(22,23)$. However, both renal portions SWV values 
were not independent predictors of $\mathrm{mAlb}+$ status when adjusted for age by multivariate analysis. Hence focal impaired elasticity in mAlb+ group as compared to mAlb- group can not be considered plausible.

The impairment of whole kidney elasticity probably requires more profound histological changes due to diabetic nephropathy. Previous studies have shown reduced global kidney SWV values along with decreased eGFR values with or without severe proteinuria $(6,12,19)$. However these findings probably require more severe kidney damage beyond glomerulosclerosis such as tubulointerstitial fibrosis and vascular changes which are deemed irreversible. We believe early recognition of disturbed kidney elasticity as a surrogate marker of early kidney damage is not possible by 2D-SWV measurement at this stage. However due to conflicting results when compared with the previous work using ARFI technique, further controlled longitudinal studies in larger series with biopsy and histopathology being used as reference standard are required in this regard.

The reproducibility of $2 \mathrm{D}-\mathrm{SWV}$ was good in the current study with an interobserver agreement ICC value of 0.66 , which is close to previous reports.

The major strength of our work is that it is the first study to evaluate the ability of novel 2D-SWV US elastography technique in revealing early kidney damage in patients with DM-II. Furthermore, application of strict inclusion criteria to eliminate the potential confounders of disturbed elasticity and proteinuria is another strength of our work. The sample size was also calculated a priori, and the number of patients included are adequate to reveal a difference between the SWV values of the study groups.

The main limitations in current study is the absence of histopathological confirmation of kidney damage. We believe, a combination of moderate albuminuria with preserved eGFR is a quite pragmatic way to reveal the early diabetic kidney damage, where biopsy is not available. Although very unlikely, absence of a nondiabetic cause of moderately increased uACR cannot be guaranteed based on exclusion of diseases by history and symptoms alone.

In conclusion, the kidney elasticity does not seem to be disturbed in patients with diabetes and a preserved eGFR with or without moderate albuminuria.

CONFLICT OF INTEREST: None.

\section{REFERENCES}

Kidney Disease: Improving Global Outcomes (KDIGO) CKD Work Group. KDIGO 2012 clinical practice guideline for the evaluation and management of chronic kidney disease. Kidney Int Suppl. 2013; 3: 1-150.

Webster AC, Nagler EV, Morton RL et al. Chronic kidney disease. Lancet. 2017; 389: 1238-52.

Schena FP, Gesualdo L. Pathogenetic mechanisms of diabetic nephropathy. J Am Soc Nephrol 2005; 16 Suppl 1: S30-S33.

Yamashita SR, von Atzingen AC, Iared W et al. Value of renal cortical thickness as a predictor of renal function impairment in chronic renal disease patients. Radiol Bras 2005; 48: 12-16.

Beland MD, Walle NL, Machan JT et al. Renal cortical thickness measured at ultrasound: is it better than renal length as an indicator of renal function in chronic kidney disease? AJR Am J Roentgenol 2010; 195: W146-149.

Lin HY, Lee YL, Lin KD et al. Association of Renal Elasticity and Renal Function Progression in Patients with Chronic Kidney Disease Evaluated by Real-Time Ultrasound Elastography. Sci Rep. 2017; 7: 43303.

White KE, Bilous RW. Type 2 diabetic patients with nephropathy show structural-functional relationships that are similar to type 1 disease. J Am Soc Nephrol. 2000; 11: 1667-73.

Walker PD, Cavallo T, Bonsib SM; Ad Hoc Committee on Renal Biopsy Guidelines of the Renal Pathology Society. Practice guidelines for the renal biopsy. Mod Pathol 2004; 17: 1555-1563. 
Sigrist RMS, Liau J, Kaffas AE et al. Ultrasound Elastography: Review of Techniques and Clinical Applications. Theranostics. 2017; 7: 1303-1329.

Cunningham A, Benediktsson H, Muruve DA et al. Trends in Biopsy-Based Diagnosis of Kidney Disease: A Population Study. Can J Kidney Health Dis. 2018; 5: 2054358118799690.

Li J, An C, Kang L et al. Recent advances in magnetic resonance imaging assessment of renal fibrosis. Adv Chronic Kidney Dis 2017; 24: 150-153.

Guo LH, Xu HX, Fu HJ et al. Acoustic radiation force impulse imaging for noninvasive evaluation of renal parenchyma elasticity: preliminary findings. PLoS One. 2013; 8: e68925.

Singh H, Panta OB, Khanal U et al. Renal Cortical Elastography: Normal Values and Variations. J Med Ultrasound. 2017; 25: 215-220.

Lee ES, Lee JB, Park HR, et al. Shear Wave Liver Elastography with a Propagation Map: Diagnostic Performance and Inter-Observer Correlation for Hepatic Fibrosis in Chronic Hepatitis. Ultrasound Med Biol. 2017; 43: 1355-1363

Ferraioli G, De Silvestri A, Lissandrin R et al. Evaluation of Inter-System Variability in Liver Stiffness Measurements. Ultraschall Med. 2019; 40: 64-75.

Levey AS, de Jong PE, Coresh J et al. The definition, classification, and prognosis of chronic kidney disease: a KDIGO Controversies Conference report. Kidney Int. 2011; 80: 17.

Goya C, Kilinc F, Hamidi C, et al. Acoustic radiation force impulse imaging for evaluation of renal parenchyma elasticity in diabetic nephropathy. AJR Am J Roentgenol. 2015;204:324-329.

Fioretto P, Mauer M, Brocco E, et al. Patterns of renal injury in NIDDM patients with microalbuminuria. Diabetologia 1996; 39: 1569-1576.

Yang H, Sun Y, Tang Y et al. Shear-wave elastography of the liver in a healthy pediatric population. J Clin Ultrasound. 2020; 48: 139-144.

Asano K, Ogata A, Tanaka K et al. l. Acoustic radiation force impulse elastography of the kidneys: is shear wave velocity affected by tissue fibrosis or renal blood flow? J Ultrasound Med 2014;33:793-801.

Bob F, Grosu I, Sporea I et al. Ultrasound-Based Shear Wave Elastography in the Assessment of Patients with Diabetic Kidney Disease. Ultrasound Med Biol. 2017; 43: 2159-216.

Ekinci EI, Jerums G, Skene A et al. Renal structure in normoalbuminuric and albuminuric patients with type 2 diabetes and impaired renal function. Diabetes Care. 2013; 36: 3620-6.

Fioretto P, Mauer M. Diabetic nephropathy: diabetic nephropathy-challenges in pathologic classification. Nat Rev Nephrol. 2010; 6 :508-510.

\section{Hosted file}

Table 1.docx available at https://authorea.com/users/417748/articles/524739-doesdiabetic-early-kidney-damage-alter-renal-elasticity-an-ultrasound-based-2d-shear-waveelastography-study

\section{Hosted file}

Table 2.docx available at https://authorea.com/users/417748/articles/524739-doesdiabetic-early-kidney-damage-alter-renal-elasticity-an-ultrasound-based-2d-shear-waveelastography-study

\section{Hosted file}


Table 3.docx available at https://authorea.com/users/417748/articles/524739-doesdiabetic-early-kidney-damage-alter-renal-elasticity-an-ultrasound-based-2d-shear-waveelastography-study

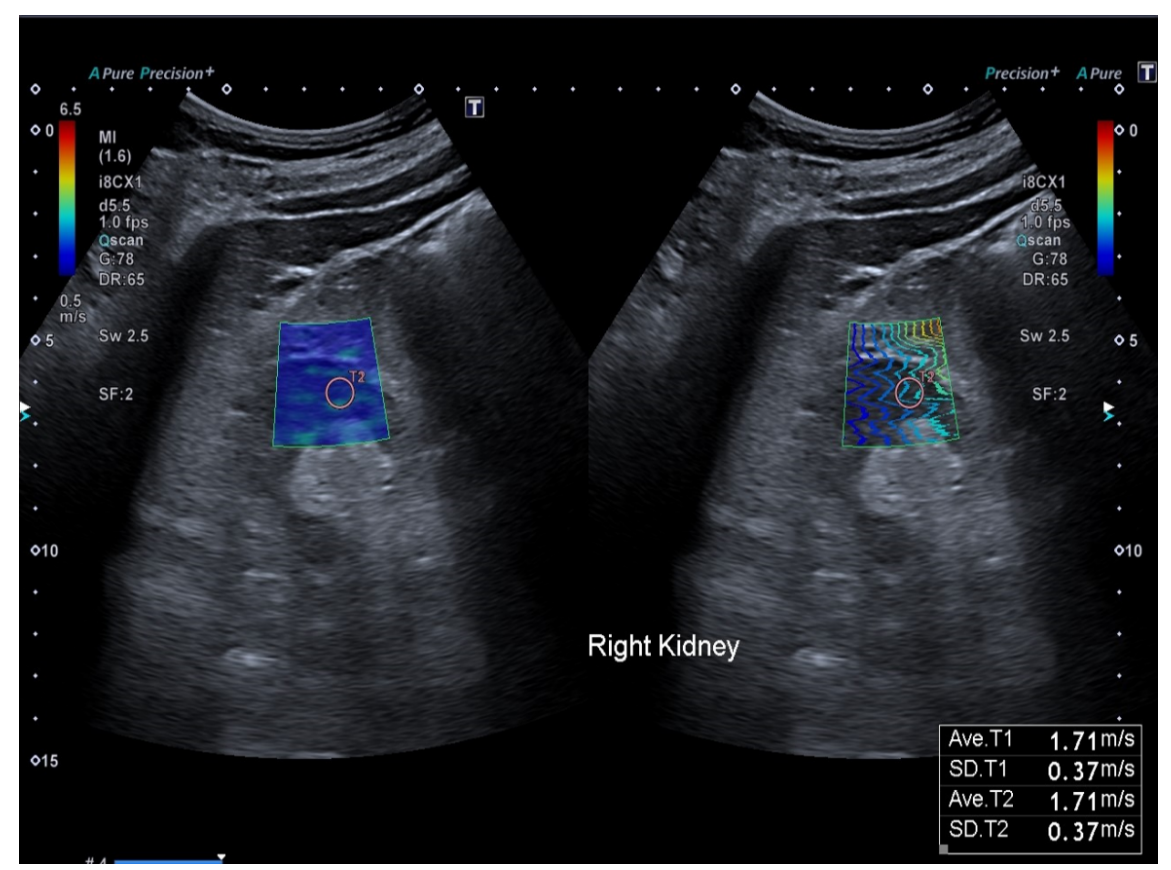

